\title{
Endometrial Cancer: When Upfront Surgery Is Not an Option
}

\author{
Amelia Barcellini $^{a} \quad$ Marianna Roccio $^{b}$ Concetta Lalisciac ${ }^{c}$ Francesca Zanellini $^{b}$ \\ Diana Pettinato $^{b}$ Francesca Valvo $^{a}$ Alfredo Mirandola ${ }^{a}$ Ester Orlandi ${ }^{a}$ \\ Angiolo Gadducci ${ }^{\text {d }}$

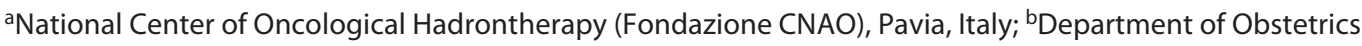 \\ and Gynecology, Fondazione IRCCS Policlinico San Matteo and University of Pavia, Pavia, Italy; ${ }^{\circ}$ Department of

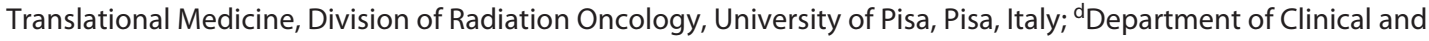 \\ Experimental Medicine, Division of Gynecology and Obstetrics, University of Pisa, Pisa, Italy
}

\section{Keywords}

Endometrial cancer · Medically unfit · Hormonotherapy ·

Chemotherapy · Brachytherapy · Hadrontherapy

\section{Abstract}

Background and Summary: The management of endometrial cancer, in an ever-older population with considerable comorbidity, remains a challenge for gynecological and radiation oncologists. Key Message: The present paper reviews literature data on treatment options for endometrial cancer patients unfit for surgery.

๑) 2020 S. Karger AG, Basel

\section{Introduction}

Endometrial cancer (EC) is the most common gynecological cancer in industrialized countries and second, after cervical cancer, in the developing world [1]. Most cases are in an apparent early stage at presentation, and laparoscopic total hysterectomy, bilateral salpingo-oophorectomy, and lymph node assessment represent the gold standard treatment in this clinical setting. Robotic surgery could be a useful alternative option to laparoscopy in severely obese women with EC [2]. Pelvic and para-aortic lymphadenectomy is useful for staging and prognosis definition, but its therapeutic function is still debated [3-6]. Both the study of Benedetti Panici et al. [3] and the ASTEC trial [7] failed to detect an improvement in disease-free survival or overall survival for women with early-stage EC treated with pelvic lymphadenectomy compared to those who were not. Anyway, patients included in these studies presented a low risk of presenting lymph node involvement to demonstrate a positive effect of the lymphadenectomy on the clinical outcome $[4,5]$. Conversely, the retrospective cohort study SEPAL [6] reported that in intermediate- and high-risk EC patients, disease-specific survival rates were higher in women who underwent pelvic plus para-aortic lymphadenectomy than in those treated with pelvic lymphadenectomy alone. These results were not confirmed for low-risk EC.

Large series suggest that sentinel lymph node mapping increases the identification of macro-metastases, micrometastases, and isolated tumor cells and does not impair oncologic outcomes compared with standard lymphadenectomy both in patients with limited myometrial invasion and in those with deeply invasive endometrioid EC karger@karger.com www.karger.com/ocl

(C) 2020 S. Karger AG, Base

Karger"
Amelia Barcellini

National Center of Oncological Hadrontherapy (Fondazione CNAO) Strada Campeggi 53

IT-27100 Pavia (Italy)

Amelia.Barcellini@cnao.it 
[8-10]. Sentinel lymph node mapping reduces operative times and improves peri-operative surgical outcomes of robotic-assisted EC staging without worsening the morbidity of hysterectomy alone [11]. An Italian multicentric and retrospective study [12], which reviewed 1,606 EC (stages I to IV) patients, reported that 209 (13\%) subjects recurred, the majority within 24 months, in the vagina $(16.7 \%)$, in the pelvis $(32.1 \%)$, and distant locations (51.2\%) [12].

Patients not fit for laparoscopic or robotic surgery may undergo vaginal hysterectomy with or without bilateral salpingo-oophorectomy also with locoregional anesthesia [13]. However, an ever-aging population with considerable comorbidities implicates that $4-9 \%$ of EC cases are unfit for surgery also by vaginal route $[14,15]$. It must be taken into consideration that obesity and diabetes are risk factors for EC and they are often related to other comorbidities (i.e., cardiovascular disease) that, as well as age, may contraindicate an upfront surgery. In the case of medically inoperable EC or in women who refuse the surgical approach, hormonotherapy (HT), radiation therapy (RT), and seldom chemotherapy (CT) may be an option, with a curative or palliative aim.

All patients' cases evaluated unfit for surgery should be discussed by a multidisciplinary team since the same concerns could also limit the feasibility of the radical brachytherapy (BT) approach [16]. No randomized studies on the comparison between RT, HT, and palliative CT have been conducted.

In March 2020, we conducted a comprehensive literature search to focus the current knowledge about the management of inoperable EC, and, especially, the potential role of particle RT.

\section{Role of HT}

Since the endocrine sex hormone correlation with the majority of ECs, specific drugs that target estrogen receptor and progesterone receptors (PR) or that inhibit estrogen synthesis have been considered and implemented for ECs of low-grade endometrioid histology [17-19]. Instead, there is little evidence that HT in any schedule (alone or in combination) improves the overall survival of women with advanced ECs [20]. HT offers a bearable and partially effective treatment for recurrent ECs, and this is the reason why an accurate patient selection is essential to have chances to succeed [21]. The main HTs for EC are represented by progestins because of their antiproliferative effects on EC cell growth. In their review,
Ehrlich et al. [22] reported a greater clinical response rate to PR-positive cancers (72\%) compared to PR-negative ones (12\%), which suggested that this treatment could be an option for selected early and PR-positive ECs [22]. Levonorgestrel-releasing intrauterine device (LNG-IUD) has a profound suppressive effect on atypical endometrial growth and represents a well-tolerated and safe option for patients who are not eligible for surgery [23]. Retrospective analyses and small case series reported clinical and pathological response rates between 60 and 80\% [23], but the relapse rate is quite high and LNG-IUD does not represent a curative option.

Tamoxifen, an anti-estrogen drug administered in the treatment and prevention of breast tumors, is known as a risk factor for EC. Paradoxically, tamoxifen has also been used in the management of metastatic ECs [24]. Gonadotropin-releasing hormone ( $\mathrm{GnRH})$ agonists are agents able to reduce estrogen levels in premenopausal women by the inhibition of pituitary and gonadal function. Moreover, a high percentage of EC cells possess receptors for $\mathrm{GnRH}$, including high-grade cancers. One study involving the treatment of EC with a GnRH agonist observed an objective response rate of $28 \%$ [25], whereas another trial failed to reveal any meaningful activity [26]. Further studies on these agents should be performed before concluding their real usefulness in this clinical setting [27]. Even in postmenopausal women, estrogens are still produced in peripheral fat tissues. Aromatase inhibitors, by blocking the conversion of androgens in estrogens, can lower circulating estrogen levels. Bellone et al. [28] reported a 58-year-old woman with recurrent EC resistant to CT but successfully treated with anastrozole. Effectiveness of anastrozole was described also in a phase II GOG study in which HT achieved a partial response in $2(8.7 \%)$ and a short-term stable disease in $2(8.7 \%)$ of 23 patients with advanced, recurrent, or persistent EC [29]. In a multicenter phase II Canadian trial, letrozole achieved a 9.4\% objective response rate and a $39.3 \%$ stable disease rate in a similar subset of 28 patients [30].

Aromatase inhibitors have significant limitations, such as toxicities and drug resistance. The development of a new generation of inhibitors with greater specificity and fewer side effects is strongly warranted for the management of EC [31]. The combination of aromatase inhibitors with mammalian target of rapamycin (m-TOR) inhibitors (such as everolimus), and of aromatase inhibitors with $\mathrm{m}$-TOR inhibitors and metformin could offer promising perspectives of clinical research [32-34]. The employment of the cyclin-dependent kinase $4 / 6$ inhibitor palbociclib in EC is still investigational [35].
Barcellini et al. 


\section{Role of CT}

Current international guidelines suggested adjuvant CT for poor-prognosis groups, including patients with stage IIIB or stage IIIC disease of any histology and patients with stage IA (with myometrial invasion), IB, II, or IIIA serous or clear cell carcinoma [36]. Multiple CTs show some efficacy in advanced EC, the most commonly investigated are platinum, anthracyclines, and taxanes (alone or in combination) [37]. The effectiveness and tolerability of carboplatin/paclitaxel regimen (as reported in GOG 209 [27] trial along with the results of several nonrandomized phase II trials [38]) make this doublet the favorite front-line CT.

Since the results regarding second-line CT are historically poor, there are few randomized studies in this clinical scenario, mainly data about second-line CT derived from nonrandomized phase II studies in which CT was administered in a metastatic setting. Paclitaxel resulted advantageous, with response rates consistently $>20 \%$, although these data are antecedent to the employment of paclitaxel as a part of first-line treatment [39-41].

However, weekly paclitaxel has shown meaningful activity in metastatic or recurrent EC previously treated with both a platinum agent and paclitaxel [42].

Other drugs (oxaliplatin, topotecan, liposomal doxorubicin, etoposide, cyclophosphamide, pemetrexed, gemcitabine, and ifosfamide) have been evaluated, but they achieve poor response rates [43-51].

In consideration of the few therapeutic options available for advanced and recurrent EC, a new therapeutic strategy appears to be offered by immunotherapy. Considering the genomic, transcriptomic, and proteomic features, the Cancer Genome Atlas Research Network (TCGA) [52] has proposed a new classification of EC in four subgroups:

- polymerase epsilon (POLE)-ultra-mutated,

- microsatellite instability-hyper-mutated (MSI-H),

- copy-number-high serous-like,

- copy-number-low microsatellite stable [53].

The potential role of the checkpoint inhibitor finds its rationale in the high mutation rate of POLE-ultra-mutated and MSI-H [54]. Anyway, further studies should consider the dominant immunosuppressive pathway for each subtype of EC, to better identify biomarkers of response.

Dostarlimab and pembrolizumab (PD-1 inhibitors) achieved a response rate from 49 to $57 \%$ in MSI-H or deficient mismatch repair (dMMR) advanced EC, respectively. The response rate for PD-L1 inhibitors was from 27 to $43 \%$, respectively [55].

Inoperable Endometrial Cancer
FDA grants hasted the approval to pembrolizumab for tissue or site agnostic use in the treatment of patients with unresectable or metastatic solid tumors, including EC, associated with MSI-H or dMMR disease [17].

The Atezolizumab Trial in Endometrial Cancer (AtTEnd) is an ongoing phase III double-blind randomized placebo-controlled trial of the PD-L1 inhibitor atezolizumab in combination with paclitaxel and carboplatin in women with advanced recurrent/endometrial cancer (EUDRACT No. 2018-001072-37) [56].

A single-arm trial of 108 patients with previously treated advanced EC showed that the combination of pembrolizumab plus lenvatinib achieved an objective response rate at 24 weeks of $38.0 \%$ in the entire cohort, of $63.6 \%$ in patients with MSI-H tumors, and of $36.2 \%$ in patients with microsatellite-stable tumors [57]. On September 17, 2019, the FDA has hastened approval to pembrolizumab plus lenvatinib for advanced EC that is not MSI-H or $\mathrm{dMMR}$ and has a disease progression following prior systemic therapy but is unfit for surgery or RT with curative intent [58].

Future strategies should explore different clinical settings and combinations of CT and RT with checkpoint inhibitors to boost immune response and improve patient outcomes.

\section{Role of RT}

In patients not suitable for surgery, RT with a combination of external RT and BT or BT alone with radical aim should be recommended. In the consensus statement from the American Brachytherapy Society, authors describe a 5-year disease-free survival ranging from 72 to $95 \%$ and a local control (LC) rate ranging from 71 to $93 \%$ [16]. Historically, low dose rate BT, 2D planning, and a point/line-based treatment planning (i.e., Madison point, point $\mathrm{My}$, point $\mathrm{S}$, and A-line) were used [14, 59-62]. However, points and line planning did not always give adequate coverage of the tumor and they were related to an increased risk of normal tissue complication due to unnecessarily high doses to organs at risk. Recently, the advancement of 3D image-based high dose rate (HDR) BT has shown excellent results in terms of LC and toxicity $[63,64]$. Overall, BT alone is recommended for grade 1 or 2 endometrioid-type EC, myometrial invasion $<50 \%$, low-volume of the disease ( $\leq 2 \mathrm{~cm})$, and available MRI local staging [16].

A combination of RT and BT is suggested for patients with high-grade disease, deep myometrial invasion, large 
volume tumor $(>2 \mathrm{~cm})$, and unavailable MRI staging but a CT-only staging $[16,60]$.

In the case of definitive BT, clinical evaluation, often under anesthesia, allows defining the type of needed applicators that could not prescind to individual patient anatomy and uterine length. Generally, the dual tandem $\mathrm{Y}$ applicator can improve lateral coverage and the triple tandem the lateral and anteroposterior coverage. However, patient compliance could be inadequate to use the aforementioned BT applicators that are associated with protracted immobilization and long-time anesthesia. It must be kept in mind that also the modified Hyman packing (another suggested BT applicator) requires the insertion of 5 to 18 capsules, as well as prolonged immobilization and protracted anesthesia. Therefore, single tandem + vaginal cylinder applicator is mostly used, more comfortable, and better tolerated in elderly and medically inoperable patients [60].

There are different BT schedules reported in the literature $[16,65]$ : when $\mathrm{BT}$ is delivered as a boost, the RT should be delivered to the pelvis (up to a total dose of 45$50.4 \mathrm{~Gy}$ in $25-28$ fractions) [16] and the suggested BT dose fractions range from $5.2 \mathrm{~Gy}$ for 4 fractions to $8.5 \mathrm{~Gy}$ for 2 fractions sequentially [65] even if other several options are described. Weitmann et al. [66] described 4-year LC of $100 \%$ and 5-year cancer-specific survival of $100 \%$ in 13 women with stage I-II inoperable EC who underwent HDR-BT alone. Promising and comparable results have also been reported in a series of patients with stage I-III disease treated with RT and sequential HDR-BT. In the most recent available data regarding an $\mathrm{RT} \pm 3 \mathrm{D}$ HDR-BT approach, the LC ranged from $91 \%$ at 2 years [67], $93 \%$ at 3 years [14], and $69 \%$ at 6 years [68] to $80 \%$ at 12 years [69]. An emerging alternative RT to the BT procedure for inoperable EC is stereotactic body RT (SBRT). Thanks to the better collimation of the RT beams, SBRT allows delivering a high dose to the target even if it could not reproduce the rapid fall-off of the dose guaranteed by a BT treatment. In the study by Kemmerer et al. [70], SBRT resulted effective and safe for early-stage patients with encouraging low toxicity rates. Jones et al. [71] carried out a dosimetric comparison study between SBRT and intracavitary HDR-BT for management of stage I-II inoperable EC demonstrating an adequate target coverage. However, even if the planned dose met the suggested dose constraints, these authors described a difference in the total dose received from surrounding healthy organs (in particular sigmoid bowel, bowel, and femoral heads) in the SBRT plans. An important physical problem that should not be underestimated in the case of RT for inoperable EC is the uterine organ motion due both to the response of treatment and filling state of the surrounding pelvic organs. In Jones's analysis [71], however, reproducibility of the volumes and planned dosimetry was demonstrated in the daily imaging controls of SBRT treatment.

To get around the low-dose bath of RT and to obtain a higher target coverage, hadrontherapy appears as a promising RT modality and an emerging choice in gynecological oncology $[72,73]$. Heavy particles, in particular, protons and carbon ions, used in hadrontherapy are characterized by dosimetric features that make them more and more interesting. Carbon ions are characterized by a high spatial selectivity which guarantees a high concentration of the dose to the target volume of RT, low dose in the opening beam door, and a rapid fall-off of the dose beyond the Bragg's peak. Consequently, carbon ion radiotherapy (CIRT) allows delivering a high dose to the target and sparing healthy tissues more than photons. Radiobiologically, carbon ions are characterized by their higher relative biological effectiveness (RBE) compared to photons, act independently from the oxygen effect or from the phase of the cell cycle, and can create clustered and difficult-to-repair DNA damage resulting in an increased probability of cell killing and better effect on radioresistant tumor tissues [74-76]. To the best of our knowledge, there is only one report regarding CIRT for inoperable EC. This is a Japanese experience aimed to evaluate the toxicity and efficacy of CIRT in the management of this subset of women thorough a pooled analysis of data from two clinical trials. All patients were judged inoperable for comorbidity, age, or refusal of surgery. CIRT was delivered to the tumor up to a total dose of 62.4-74.4 Gy (RBE) in 20 fractions and the authors established $60 \mathrm{~Gy}(\mathrm{RBE})$ as a limit dose to the intestine. To overcome intrafraction uterine motion, vaginal packing was used daily. Control of the bladder volume was provided by controlled retrograde filling via the transurethral catheter, and premedication with laxatives was recommended to patients to remedy differences in bowel organ filling. LC at 5 years was $86 \%$ and it was accompanied by 5 -year overall survival and 5 -year progressionfree survival of 68 and $64 \%$, respectively, without acute or late toxicity of grade $\geq 3$ [77].

\section{Conclusions}

In $2019,61,880$ new cases and 12,160 deaths for EC were reported worldwide [1]. Although mini-invasive total hysterectomy, bilateral salpingo-oophorectomy,
68

Oncology 2021;99:65-71 DOI: $10.1159 / 000510690$
Barcellini et al. 
and lymph node assessment are the cornerstones of treatment of EC, surgery may be sometimes unfeasible for elderly women who suffer from multiple comorbidities and are at high risk of surgical complications. Moreover, EC is a very heterogeneous disease. Reasonably, HT could be used in patients with positive estrogen receptor and/or PR. CT plays a role in advanced disease, especially with a palliative aim. Irradiation alone could be a safe and effective approach in case of an inoperable disease. Image-guided BT offers good results in terms of LC and toxicity. CIRT is a promising alternative to conventional RT in these clinical settings. A strong collaboration between oncologic gynecologists and radiation oncologists (specialized in BT and hadrontherapy) is warranted for the management of these difficult-to-cure patients.

\section{Conflict of Interest Statement}

The authors have no conflicts of interest to declare.

\section{Funding Sources}

None.

\section{Author Contributions}

A.B., M.R., A.G. wrote the draft; C.L., F.Z., D.P., F.V., A.M., E.O. critically revised the manuscript.

\section{References}

1 Siegel RL, Miller KD, Jemal A. Cancer statistics, 2019. CA Cancer J Clin. 2019;69(1):7-34.

2 Corrado G, Vizza E, Cela V, et al. Laparoscopic versus robotic hysterectomy in obese and extremely obese patients with endometrial cancer: A multi-institutional analysis. Eur J Surg Oncol J Eur Soc Surg Oncol Br Assoc Surg Oncol. 2018;44(12):1935-41.

3 Benedetti Panici P, Basile S, Maneschi F, et al. Systematic pelvic lymphadenectomy vs. no lymphadenectomy in early-stage endometrial carcinoma: randomized clinical trial. J Natl Cancer Inst. 2008;100(23):1707-16.

4 Hakmi A. Lymphadenectomy in endometrial cancer. Lancet (London, England). 2009; 373(9670):1169.

5 Amant F, Neven P, Vergote I. Lymphadenectomy in endometrial cancer. Lancet (London, England). 2009;373(9670):1161-9.

6 Todo Y, Kato H, Kaneuchi M, Watari H, Takeda M, Sakuragi N. Survival effect of paraaortic lymphadenectomy in endometrial cancer (SEPAL study): a retrospective cohort analysis. Lancet (London, England). 2010; 375(9721):1165-72.

7 Efficacy of systematic pelvic lymphadenectomy in endometrial cancer (MRC ASTEC trial): a randomised study. Lancet. 2009; 373(9658):125-36.

8 Zahl Eriksson AG, Ducie J, Ali N, et al. Comparison of a sentinel lymph node and a selective lymphadenectomy algorithm in patients with endometrioid endometrial carcinoma and limited myometrial invasion. Gynecol Oncol. 2016;140(3):394-9.

9 Ducie JA, Eriksson AGZ, Ali N, et al. Comparison of a sentinel lymph node mapping algorithm and comprehensive lymphadenectomy in the detection of stage IIIC endometrial carcinoma at higher risk for nodal disease. Gynecol Oncol. 2017;147(3):541-8.
10 Schlappe BA, Weaver AL, Ducie JA, et al. Multicenter study comparing oncologic outcomes between two nodal assessment methods in patients with deeply invasive endometrioid endometrial carcinoma: A sentinel lymph node algorithm versus a comprehensive pelvic and paraaortic lymphadenectomy. Gynecol Oncol. 2018;151(2):235-42.

11 Casarin J, Multinu F, Tortorella L, et al. Sentinel lymph node biopsy for robotic-assisted endometrial cancer staging: further improvement of perioperative outcomes. Int J Gynecol Cancer. 2020;30(1):41-7.

12 Sartori E, Laface B, Gadducci A, et al. Factors influencing survival in endometrial cancer relapsing patients: a Cooperation Task Force (CTF) study. Int J Gynecol Cancer. 2003; 13(4):458-65

13 Kruse A-J, Ter Brugge HG, de Haan HH, Van Eyndhoven HW, Nijman HW. Vaginal hysterectomy with or without bilateral salpingooophorectomy may be an alternative treatment for endometrial cancer patients with medical co-morbidities precluding standard surgical procedures: a systematic review. Int $\mathrm{J}$ Gynecol Cancer. Jan 18:ijgc-2018-000015. Epub ahead of print.

14 Coon D, Beriwal S, Heron DE, et al. Highdose-rate Rotte "Y" applicator brachytherapy for definitive treatment of medically inoperable endometrial cancer: 10 -year results. Int $J$ Radiat Oncol Biol Phys. 2008;71(3):779-83.

15 Nguyen TV, Petereit DG. High-dose-rate brachytherapy for medically inoperable stage I endometrial cancer. Gynecol Oncol. 1998; 71(2):196-203.

16 Schwarz JK, Beriwal S, Esthappan J, et al. Consensus statement for brachytherapy for the treatment of medically inoperable endometrial cancer. Brachytherapy. 2015;14(5):587-99.
17 Makker V, Green AK, Wenham RM, Mutch D, Davidson B, Miller DS. New therapies for advanced, recurrent, and metastatic endometrial cancers. Gynecol Oncol Res Pract. 2017;4:19.

18 Varga A, Henriksen E. Clinical and histopathologic evaluation of the effect of 17-alpha-hydroxyprogesterone-17-n-caproate on endometral carcinoma. Obstet Gynecol. 1961;18: 658-72.

19 Gotlieb WH, Beiner ME, Shalmon B, et al Outcome of fertility-sparing treatment with progestins in young patients with endometrial cancer. Obstet Gynecol. 2003;102(4):718-25.

20 Kokka F, Brockbank E, Oram D, Gallagher C, Bryant A. Hormonal therapy in advanced or recurrent endometrial cancer. Cochrane $\mathrm{Da}$ tabase Syst Rev. 2010;12:CD007926.

21 Carlson MJ, Thiel KW, Leslie KK. Past, present, and future of hormonal therapy in recurrent endometrial cancer. Int $J$ Womens Health. 2014;6:429-35.

22 Ehrlich CE, Young PC, Stehman FB, Sutton GP, Alford WM. Steroid receptors and clinical outcome in patients with adenocarcinoma of the endometrium. Am J Obstet Gynecol. 1988;158(4):796-807.

23 Pal N, Broaddus RR, Urbauer DL, et al. Treatment of Low-Risk Endometrial Cancer and Complex Atypical Hyperplasia With the Levonorgestrel-Releasing Intrauterine Device. Obstet Gynecol. 2018;131(1):109-16.

24 Thigpen T, Brady MF, Homesley HD, Soper JT, Bell J. Tamoxifen in the treatment of advanced or recurrent endometrial carcinoma: a Gynecologic Oncology Group study. J Clin Oncol. 2001;19(2):364-7.

25 Jeyarajah AR, Gallagher CJ, Blake PR, et al. Long-term follow-up of gonadotrophin-releasing hormone analog treatment for recurrent endometrial cancer. Gynecol Oncol. 1996;63(1):47-52. 
26 Covens A, Thomas G, Shaw P, et al. A phase II study of leuprolide in advanced/recurrent endometrial cancer. Gynecol Oncol. 1997; 64(1):126-9.

27 Markman M. Hormonal therapy of endometrial cancer. Eur J Cancer. 2005;41(5):673-5.

28 Bellone S, Shah HR, McKenney JK, Stone PJB, Santin AD. Recurrent endometrial carcinoma regression with the use of the aromatase inhibitor anastrozole. Am J Obstet Gynecol. 2008;199(3):e7-e10.

29 Rose PG, Brunetto VL, VanLe L, Bell J, Walker JL, Lee RB. A phase II trial of anastrozole in advanced recurrent or persistent endometrial carcinoma: a Gynecologic Oncology Group study. Gynecol Oncol. 2000;78(2):212-6.

30 Ma BBY, Oza A, Eisenhauer E, et al. The activity of letrozole in patients with advanced or recurrent endometrial cancer and correlation with biological markers - a study of the $\mathrm{Na}$ tional Cancer Institute of Canada Clinical Trials Group. Int J Gynecol Cancer. 2004; 14(4):650-8.

31 Gao C, Wang Y, Tian W, Zhu Y, Xue F. The therapeutic significance of aromatase inhibitors in endometrial carcinoma. Gynecol Oncol. 2014;134(1):190-5.

32 Soliman PT, Westin SN, Iglesias DA, et al. Everolimus, Letrozole, and Metformin in Women with Advanced or Recurrent Endometrioid Endometrial Cancer: A Multi-Center, Single Arm, Phase II Study. Clin Cancer Res. 2020;26(3):581-7.

33 Wheler JJ, Moulder SL, Naing A, et al. Anastrozole and everolimus in advanced gynecologic and breast malignancies: activity and molecular alterations in the PI3K/AKT/ mTOR pathway. Oncotarget. 2014;5(10): 3029-38.

34 Slomovitz BM, Jiang Y, Yates MS, et al. Phase II study of everolimus and letrozole in patients with recurrent endometrial carcinoma. J Clin Oncol. 2015;33(8):930-6.

35 Tanaka T, Terai Y, Ashihara K, et al. The efficacy of the cyclin-dependent kinase $4 / 6$ inhibitor in endometrial cancer. PLoS One. 2017;12(5):e0177019.

36 Koh W-J, Abu-Rustum NR, Bean S, et al Uterine Neoplasms, Version 1.2018, NCCN Clinical Practice Guidelines in Oncology. J Natl Compr Canc Netw. 2018;16(2):170-99.

37 Humber CE, Tierney JF, Symonds RP, et al. Chemotherapy for advanced, recurrent or metastatic endometrial cancer: a systematic review of Cochrane collaboration. Ann Oncol. 2007;18(3):409-20.

38 Bestvina CM, Fleming GF. Chemotherapy for Endometrial Cancer in Adjuvant and Advanced Disease Settings. Oncologist. 2016; 21(10):1250-9.

39 Homesley HD, Meltzer NP, Nieves L, Vaccarello L, Lowendowski GS, Elbendary AA. A phase II trial of weekly 1-hour paclitaxel as second-line therapy for endometrial and cervical cancer. Int J Clin Oncol. 2008;13(1):625.
40 Lincoln S, Blessing JA, Lee RB, Rocereto TF. Activity of paclitaxel as second-line chemotherapy in endometrial carcinoma: a Gynecologic Oncology Group study. Gynecol Oncol. 2003;88(3):277-81.

41 Lissoni A, Zanetta G, Losa G, Gabriele A, Parma G, Mangioni C. Phase II study of paclitaxel as salvage treatment in advanced endometrial cancer. Ann Oncol. 1996;7(8):861-3.

42 Markman M, Fowler J. Activity of weekly paclitaxel in patients with advanced endometrial cancer previously treated with both a platinum agent and paclitaxel. Gynecol Oncol. 2004;92(1):180-2.

43 Fracasso PM, Blessing JA, Molpus KL, Adler LM, Sorosky JI, Rose PG. Phase II study of oxaliplatin as second-line chemotherapy in endometrial carcinoma: a Gynecologic Oncology Group study. Gynecol Oncol. 2006; 103(2):523-6.

44 Gupta D, Owers RL, Kim M, et al. A phase II study of weekly topotecan and docetaxel in heavily treated patients with recurrent uterine and ovarian cancers. Gynecol Oncol. 2009; 113(3):327-30

45 Miller DS, Blessing JA, Lentz SS, Waggoner SE. A phase II trial of topotecan in patients with advanced, persistent, or recurrent endometrial carcinoma: a gynecologic oncology group study. Gynecol Oncol. 2002;87(3):24751.

46 Muggia FM, Blessing JA, Sorosky J, Reid GC. Phase II trial of the pegylated liposomal doxorubicin in previously treated metastatic endometrial cancer: a Gynecologic Oncology Group study. J Clin Oncol. 2002;20(9):23604.

47 Rose PG, Blessing JA, Lewandowski GS Creasman WT, Webster KD. A phase II trial of prolonged oral etoposide (VP-16) as second-line therapy for advanced and recurrent endometrial carcinoma: a Gynecologic Oncology Group study. Gynecol Oncol. 1996; 63(1):101-4

48 Pawinski A, Tumolo S, Hoesel G, et al. Cyclophosphamide or ifosfamide in patients with advanced and/or recurrent endometrial carcinoma: a randomized phase II study of the EORTC Gynecological Cancer Cooperative Group. Eur J Obstet Gynecol Reprod Biol. 1999;86(2):179-83.

49 Miller DS, Blessing JA, Drake RD, et al. A phase II evaluation of pemetrexed (Alimta, LY231514, IND \#40061) in the treatment of recurrent or persistent endometrial carcinoma: a phase II study of the Gynecologic Oncology. Gynecol Oncol. 2009;115(3):443-6.

50 Tait DL, Blessing JA, Hoffman JS, et al. A phase II study of gemcitabine (gemzar, LY188011) in the treatment of recurrent or persistent endometrial carcinoma: a gynecologic oncology group study. Gynecol Oncol. 2011;121(1):118-21.
51 Sutton GP, Blessing JA, Homesley HD, McGuire WP, Adcock L. Phase II study of ifosfamide and mesna in refractory adenocarcinoma of the endometrium. A Gynecologic Oncology Group study. Cancer. 1994;73(5): 1453-5.

52 Levine DA, Getz G, Gabriel SB, et al. Integrated genomic characterization of endometrial carcinoma. Nature. 2013;497(7447):67-73.

53 Douglas JB, Silverman DT, Pollak MN, Tao Y, Soliman AS, Stolzenberg-Solomon RZ. Serum IGF-I, IGF-II, IGFBP-3, and IGF-I/ IGFBP-3 Molar Ratio and Risk of Pancreatic Cancer in the Prostate, Lung, Colorectal, and Ovarian Cancer Screening Trial. Cancer Epidemiol Biomarkers Prev. 2010;19(9):2298306.

54 Mittica G, Ghisoni E, Giannone G, Aglietta M, Genta S, Valabrega G. Checkpoint inhibitors in endometrial cancer: preclinical rationale and clinical activity. Oncotarget. 2017; 8(52):90532-44.

55 Green AK, Feinberg J, Makker V. A Review of Immune Checkpoint Blockade Therapy in Endometrial Cancer. Am Soc Clin Oncol Educ book Am Soc Clin Oncol Annu Meet. 2020;40:1-7.

$56 \mathrm{https} / / / \mathrm{www} . c l i n i c a l t r i a l s r e g i s t e r . e u / c t r-$ search/search?query $=2018-001072-37$.

57 Makker V, Taylor MH, Aghajanian C, et al Lenvatinib Plus Pembrolizumab in Patients With Advanced Endometrial Cancer. J Clin Oncol. 2020 Sep 10;38(26):2981-92.

58 Arora S, Balasubramaniam S, Zhang W, et al FDA Approval Summary: Pembrolizumab plus Lenvatinib for Endometrial Carcinoma, a Collaborative International Review under Project Orbis. Clin Cancer Res. Published online April 2020.

59 https://www.acr.org/-/media/ACR/Files/ Practice-Parameters/RadOnc.pdf.

60 Montemaggi P, Trombetta M, Brady L (eds). Brachytherapy: An International Perspective. Cham, Springer International Publishing Switzerland, 2016.

61 Stitt JA, Fowler JF, Thomadsen BR, Buchler DA, Paliwal BP, Kinsella TJ. High dose rate intracavitary brachytherapy for carcinoma of the cervix: the Madison system: I. Clinical and radiobiological considerations. Int J Radiat Oncol Biol Phys. 1992;24(2):335-48.

62 Knocke TH, Kucera H, Weidinger B, Höller W, Pötter R. Primary treatment of endometrial carcinoma with high-dose-rate brachytherapy: results of 12 years of experience with 280 patients. Int J Radiat Oncol Biol Phys. 1997;37(2):359-65

63 Barcellini A, Lecchi M, Tenconi C, et al. Highdose-rate brachytherapy for high-grade vaginal intraepithelial neoplasia: A dosimetric analysis. J Contemp Brachytherapy. 2019; $11(2)$

64 Delishaj D, Barcellini A, D'Amico R, et al Vaginal toxicity after high-dose-rate endovaginal brachytherapy: 20 years of results. J Contemp Brachytherapy. 2018;10(6). 
65 Nag S, Erickson B, Parikh S, Gupta N, Varia M, Glasgow G. The American Brachytherapy Society recommendations for high-dose-rate brachytherapy for carcinoma of the endometrium. Int J Radiat Oncol Biol Phys. 2000; 48(3):779-90.

66 Weitmann HD, Pötter R, Waldhäusl C, Nechvile E, Kirisits C, Knocke TH. Pilot study in the treatment of endometrial carcinoma with 3D image-based high-dose-rate brachytherapy using modified Heyman packing: clinical experience and dose-volume histogram analysis. Int J Radiat Oncol Biol Phys. 2005;62(2): 468-78.

67 Gill BS, Kim H, Houser C, et al. Image-based three-dimensional conformal brachytherapy for medically inoperable endometrial carcinoma. Brachytherapy. 2014;13(6):542-7.

68 Draghini L, Maranzano E, Casale M, et al. Definitive three-dimensional high-dose-rate brachytherapy for inoperable endometrial cancer. J Contemp Brachytherapy. 2017;9(2): $118-23$.
69 Acharya S, Perkins SM, DeWees T, et al. Brachytherapy Is Associated With Improved Survival in Inoperable Stage I Endometrial Adenocarcinoma: A Population-Based Analysis. Int J Radiat Oncol Biol Phys. 2015;93(3): 649-57.

70 Kemmerer E, Hernandez E, Ferriss JS, et al. Use of image-guided stereotactic body radiation therapy in lieu of intracavitary brachytherapy for the treatment of inoperable endometrial neoplasia. Int J Radiat Oncol Biol Phys. 2013;85(1):129-35.

71 Jones R, Chen Q, Best R, Libby B, Crandley EF, Showalter TN. Dosimetric feasibility of stereotactic body radiation therapy as an alternative to brachytherapy for definitive treatment of medically inoperable early stage endometrial cancer. Radiat Oncol. 2014;9: 164.

72 Wang L, Wang X, Zhang Q, et al. Is there a role for carbon therapy in the treatment of gynecological carcinomas? A systematic review. Future Oncol. 2019;15(26):3081-95.
73 Barcellini A, Gadducci A, Laliscia C, et al. Adenoid Cystic Carcinoma of Bartholin's Gland: What Is the Best Approach? Oncology. 2020; 98(8):513-9.

74 Barcellini A, Vitolo V, Facoetti A, et al. Feasibility of carbon ion radiotherapy in the treatment of gynecological melanoma. In Vivo (Brooklyn). 2019;33(2):473-6.

75 Facoetti A, Barcellini A, Valvo F, Pullia M. The Role of Particle Therapy in the Risk of Radio-induced Second Tumors: A review of the literature. Anticancer Res. 2019;39(9).

76 Barcellini A, Vitolo V, Mastella E, Mirandola A, Valvo F. Letter to the Editor concerning "Re-irradiation in gynaecological cancers, present experiences and future hopes." J Radiat Oncol. 2019;8(3):355-6.

77 Irie D, Okonogi N, Wakatsuki M, et al. Carbon-ion radiotherapy for inoperable endometrial carcinoma. J Radiat Res. 2018;59(3): 309-15. 